\title{
A SEARCH FOR PULSED HARD RADIATION FROM THE CRAB.NEBULA
}

\author{
G. J. FISHMAN, F. R. HARNDEN, Jr., and R. C. HAYMES \\ Dept. of Space Science, William Marsh Rice University, Houston, Tex., U.S.A.
}

This paper reports the detection of repetitive pulses of hard X-rays and gamma rays in data obtained in mid-1967 from the Crab Nebula. The pulses are believed to originate from NP0532 since the observed repetition period is what would be expected on the basis of an extrapolation of recent optical data obtained for NP0532 back to the time of the observation.

The experiment reported here was conducted at balloon altitudes on June 4, 1967 and has been previously described (Haymes et al., 1968). Briefly, the actively-collimated $\gamma$-ray detector has a field of view characterized by a half-flux angle of $12^{\circ}$ from the axis. Its associated pulse height analyzer was arranged to detect and analyze those photons having energies greater than $35 \mathrm{keV}$.

While the balloon floated at approximately 130000 feet altitude, data were collected for $3 \frac{1}{2}$ hours; for 10 -min intervals the detector was pointed at the Crab (to within $\pm 1^{\circ}$ ) and alternately at a background region offset by $180^{\circ}$ in azimuth. In the present analysis, we have searched $80 \mathrm{~min}$ of source data, and for reference and control purposes, $30 \mathrm{~min}$ of background data, for hard X-ray pulsations.

The detection of each photon was telemetered to ground receiving stations where it was recorded on analog magnetic tape along with time signals received from WWV.

The time at which each photon event occurred has been determined using WWV as a time base. Short-term speed fluctuations of the tape recorder were found to be less than $\frac{1}{10}$ of a millisec, peak to peak, in a 1-sec time interval. A delay time, introduced by the balloon-borne system, between the detection and recording of an event consists of a constant and a variable component. The constant component merely introduces a phase shift ( $\sim 2$ millisec) and does not affect the present analysis. The variable delay is due mainly to the photon-energy dependence of the pulse height analysis time. For approximately one percent of the events, this delay may be as large as $\mathbf{0 . 5}$ millisec; otherwise, it is less than 0.2 millisec.

In the present analysis, the apparent time of arrival of each photon was measured with 1.0 millisec resolution. The nearest millisec interpolation between the 1-sec pulses from WWV was accomplished with an Astrodata Corporation Model 6190 time-code generator, which has a time base stable to one part in $10^{8}$. The times of photon arrivals and of the $1-\mathrm{sec}$ WWV pulses, as measured by the time-code generator, were recorded on digital tape.

The slow-down rate for NP0532, determined optically by Warner et al. (1969), was used in a linear 575-day extrapolation from their measurements to the time of the flight. The extrapolation resulted in a predicted heliocentric period ' $P_{0}$ ' of $33.071758 \pm$ \pm 0.000047 millisec at $0930 \mathrm{CDT}$ on the morning of the flight. With the aid of a 
digital computer, searches for periodicity were made over $1.4 \times 10^{5}$ NP0532 periods, using a superposed epoch analysis. Over 200 searches were made at periods differing by 5 nanosec increments and covering a 1200 nanosec range which included the geocentric period corresponding to $P_{0}$. Each search determined the number of counts per millisec for each millisec of phase.

During the search at each assumed geocentric period (figured for an epoch at 0930 CDT), the value of the period was updated at 20-min intervals to account for the apparent change in the period of NP0532. The effects for which corrections were made throughout the flight, in order of their importance, are: the change in the diurnal radial velocity component; the change in the earth's orbital radial velocity component; the intrinsic slow-down of NP0532; and the balloon drift motion (change in propagation delay). The above terms had the net effect of increasing the apparent period of NP0532 by 18 nanosec over the 190-min interval during which the 80 min of data were obtained.

The diurnal and orbital radial velocity terms were calculated for the position of the south-preceding central star of the Crab Nebula as given by Minkowski (1968). This object has been identified as the source of the optical pulsations (Cocke et al., 1969; Lynds et al., 1969).

For a barycentric period of 33.071783 millisec, one peak at the $3.9 \sigma(\sigma=$ standard deviation) level and another, $14 \mathrm{msec}$ later in phase, at the $3.0 \sigma$ level were detected. This was the only period that resulted in a peak exceeding 3 standard deviations above the mean number of counts. Observation of the interpulse with the expected phase delay considerably enhances the statistical significance of the period determination. The period was then varied about this value in 1-nanosec increments. No further peak increases were observed, nor did any additional structural features emerge. It appears that the barycentric period has been established to \pm 10 nanosec since shifting the period by this amount reduces the peaks to $\sim 2$ standard deviations.

In Figure 1 the resulting pulse profile is shown along with the optical data of Lynds et al. (1969). A double-pulse structure rather like that found at optical wavelengths is evident; 14 millisec separate the interpulse from the main pulse. The interpulse is somewhat wider than the main pulse, which is also true for the optical pulse profile. It may be significant that the power contained in the interpulse seems nearly equal to that in the main pulse in the energy region above $35 \mathrm{keV}$ and therefore relatively greater than it is in the optical. If it is statistically significant, this may be evidence for either a temporal variation or a wavelength-dependence of the interpulse structure. In the soft X-ray region (1-10 keV), Fritz et al. (1969) have also observed the interpulse to be stronger, relative to the main pulse, than has been observed in the optical region. This seems to support the wavelength-dependence of the pulse profile, but additional observations are required in both the soft and hard $\mathrm{X}$-ray regions.

The value found for the period is only 25 nanosec greater than $P_{0}$, a discrepancy that is well within the range of periods allowed by the uncertainties in the radio and optical slow-down rates. This suggests that there have been no discontinuous changes in the period of NP0532 between June 4, 1967 and Februari 22, 1969 of the type 
observed in the Vela pulsar, PSR 0833-45 (Radhakrishnan and Manchester, 1969; Reichley and Downs, 1969).

The present early detection of the pulsar permits an improved estimate of the slowdown rate to be made. Using recent period data (Warner et al., 1969), we calculate a linear coefficient of $36.51 \pm 0.02 \mathrm{nsec} \mathrm{day}^{-1}$ over the 575-day interval, which compares

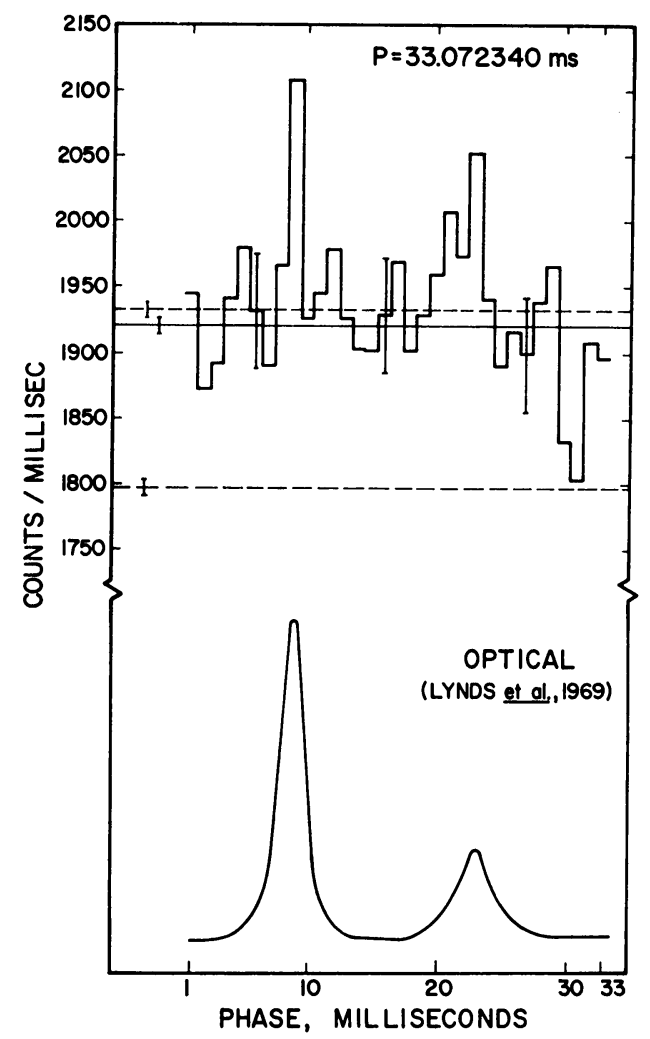

Fig. 1. Pulse profile found for photon energies greater than $35 \mathrm{keV}$ from pulsar NP0532 on June 4, 1967. The lower dashed line indicates the mean contribution from the background. The upper dashed line is the mean count rate; the solid horizontal litîe is the mean without peaks. The error bars shown are \pm 1 standard deviation. The value shown for $P$ is the geocentric period at $0930 \mathrm{CDT}$ and the optical data are shown for comparison purposes.

with the McDonald Observatory value of $36.55 \pm 0.08 \mathrm{nsec} \mathrm{day}^{-1}$ and with the Arecibo value (Richards and Comella, 1969) of $36.48 \pm 0.04 \mathrm{nsec} \mathrm{day}^{-1}$. The agreement of the above linear coefficients over the long time interval between measurements allows an upper limit of $3 \times 10^{-4} \mathrm{nsec} \mathrm{day}^{-2}$ to be placed on the magnitude of the second order term, $\left|\mathrm{d}^{2} P / \mathrm{d} t^{2}\right|$ at the $2 \sigma$ level.

Figure 2 shows the spectrum of the entire Nebula and of the pulsed component in various energy regions. Since all 128 channels of the pulse height analyzer were combined for the present analysis, no spectral information is yet available. In plotting 
the point representing the present analysis at $120 \mathrm{keV}$, we have assumed that the pulsed spectrum is like that of the hard radiation from the Nebula. $120 \mathrm{keV}$ is the geometric mean of the energies (35-560 keV) over which the Nebula's spectrum was previously determined (Haymes et al., 1968).

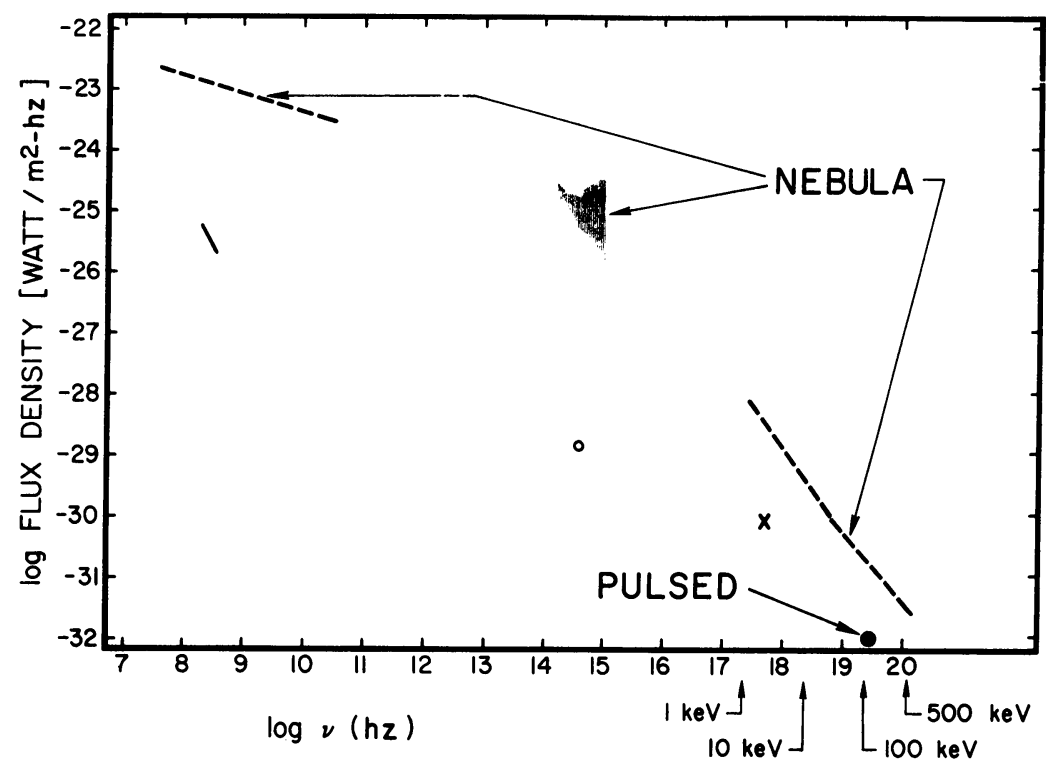

Fig. 2. Tentative spectrum for NP0532. The Nebula spectra are from Haymes et al. (1968). The pulsed radio spectrum is from Richards and Comella (1969); the optical point, from Lynds et al. (1969). The soft X-ray point $(X)$ is due to Fritz et al. (1969) and the present measurement is shown at $120 \mathrm{keV}$ under the assumption that the spectral shape is like that of the Nebula above $35 \mathrm{keV}$.

With this assumption, the $X$ - and $\gamma$-ray pulsed intensity is $7 \pm 2 \%$ of the Crab Nebula. This is equivalent to an average pulsed power of $1.6 \pm 0.4 \times 10^{-9} \mathrm{ergs} \mathrm{cm}^{-2}$ $\mathrm{sec}^{-1}$ at the top of the earth's atmosphere; the hard X- and $\gamma$-ray regions contain a major component of the pulsed luminosity of NP0532 if the spectral index is like that of the Nebula as a whole. The percentage of pulsed luminosity is of the same order as that observed in the soft X-ray region (Fritz et al., 1969), indicating a softening of the pulsed spectrum between the optical and the hard X-ray regions.

\section{Acknowledgements}

Thanks are due to Professors F. C. Michel and H. C. Goldwire for their advice, encouragement and aid in the calculations.

The research was supported in part by the Air Force Office of Scientific Research, Office of Aerospace Research, United States Air Force, under Contract No. F4462069-C-0083. 


\section{References}

Cocke, W. J., Disney, M. J., and Taylor, D. J.: 1969, Nature 221, 525.

Comella, J. M., Craft, H. D., Jr., Lovelace, R. V. E., Sutton, J. M., and Tyler, G. L.: 1969, Nature 221, 453.

Fritz, G., Henry, R. C., Meekins, J. F., Chubb, T. A., and Friedman, H.: 1969, Science 164, 709. Haymes, R. C., Ellis, D. V., Fishman, G. J., Kurfess, J. D., and Tucker, W. H.: 1968, Astrophys. J. 151, L9.

Lynds, R., Maran, S. P., and Trumbo, D. E.: 1969, Astrophys. J. 155, L121.

Minkowski, R.: 1968, in Nebulae and Interstellar Matter (ed. by B. M. Middlehurst and L. H. Aller), University of Chicago Press, Chicago, p. 639.

Radhakrishnan, V. and Manchester, R. N.: 1969, Nature 222, 228.

Reichley, P. E. and Downs, G. S.: 1969, Nature 222, 229.

Richards, D. and Comella, J. M.: 1969, Nature (preprint).

Warner, B., Nather, R. E., and MacFarlane, M.: 1969, Nature 222, 233. 